\title{
A micromachined 2D positioner with electrothermal actuation and sub-nanometer capacitive sensing
}

\author{
Larry L Chu ${ }^{1}$ and Yogesh B Gianchandani ${ }^{1,2}$ \\ ${ }^{1}$ Department of Electrical and Computer Engineering, University of Wisconsin, \\ Madison, WI, USA \\ ${ }^{2}$ EECS Department, University of Michigan, Ann Arbor, MI, USA
}

Received 10 September 2002, in final form 17 December 2002

Published 17 January 2003

Online at stacks.iop.org/JMM/13/279

\begin{abstract}
This paper reports on a multi-purpose two-axis micropositioner with sub-nanometer position sensing for precise feedback control. Along each axis it has an electrothermal actuator, a capacitive position sensor and a displacement amplifier that provides a gain of 3.37 for the sensor. It is fabricated from custom SOI wafers using dry etching, and each component is electrically and thermally isolated by silicon nitride. For a fabricated device of $65 \mu \mathrm{m}$ thickness, the measured displacement sensitivity is $0.333 \mathrm{fF} \mathrm{nm}^{-1}$, which corresponds to $0.3 \mathrm{~nm}$ resolution with available laboratory instrumentation. The range is $\approx 19 \mu \mathrm{m}$ along each axis for the positioner, which corresponds to $66 \mu \mathrm{m}$ travel in the sense combs. Using an external parallel inductor, a positioning displacement of $9.6 \mu \mathrm{m}$ offers a shift of $240 \mathrm{kHz}$ in $L-C$ resonance, corresponding to a sensitivity of $25 \mathrm{~Hz} \mathrm{~nm}^{-1}$.
\end{abstract}

(Some figures in this article are in colour only in the electronic version)

\section{Introduction}

Micropositioners have been constructed in the past using various actuators such as electrostatic [1], magnetic [2,3] and thermal transduction $[4,5]$, amongst others. Positioners are needed for many applications such as scanning microscopy, optical switching, wireless communications and data storage. Due to variations in manufacturing and operating conditions, high precision positioners must be operated with feedback control. Ultimately, the resolution of a feedback controlled positioner is limited by its ability to sense location in a precise manner over a wide dynamic range. Although some system applications provide this capability with secondary means of position sensing, most do not. While a $100 \mathrm{~nm}$ resolution positioner with built-in position sensing was reported in [2], this was a manually assembled metal device with one axis of motion. This paper describes a batch fabricated Si device with two axes (2D) of motion and sub-nanometer resolution ${ }^{3}$.

The multi-purpose micropositioner described in this work operates in-plane, meaning that the motions are parallel

3 A portion of these results have been published in conference abstract form in [6]. to the substrate. Along each positioning axis is included an electrothermal actuator [5], a compliant displacement amplifier [7] and a capacitive sensor bank. Shared elements include a sample stage, and lead transfer points. These components are shown in a schematic drawing in figure 1 . The displacement of the sample stage is amplified by the compliant structure and sensed by the capacitive banks. Since these are used exclusively for sensing, they can be densely packed without fear of electromechanical instability. The compliant mechanism does not undergo mechanical loading at the capacitive sensor end because no net electrostatic force is exerted during sensing. The signals at the sample stage can be transferred off-chip using the interconnect spring and pad. The sample stage is electrically isolated from the actuator, which is isolated from the amplifier and sensors. The two-dimensional micropositioner has two symmetrical actuation mechanisms arranged orthogonally. The two mechanisms are connected to the tip area with a long shank. The isolation plugs shown in figure 1 electrically separate the sample stage, the actuator and the position sensor.

The electrothermal bent-beam actuator is an excellent transducer of strain to displacement [8]. Joule heating in 


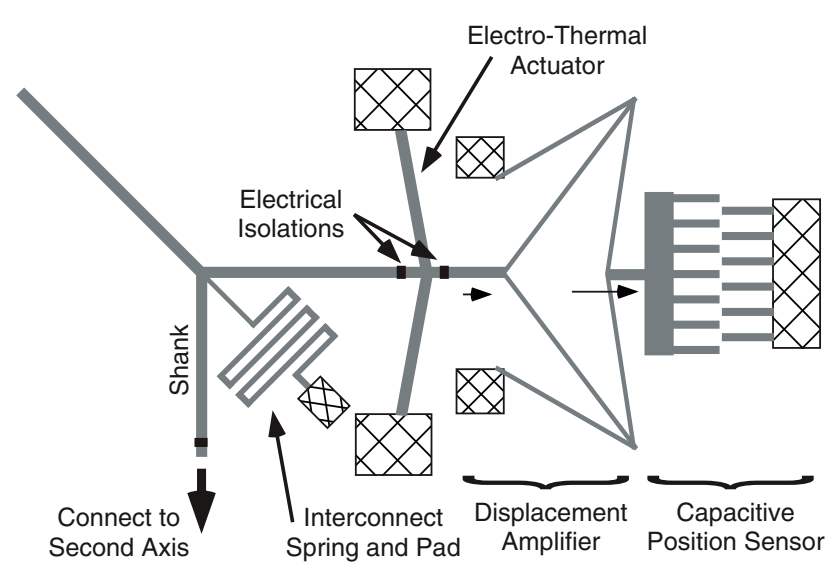

Figure 1. Schematic drawing of a $2 \mathrm{D}$ positioner showing the actuator, the amplifier and the capacitive sensor.
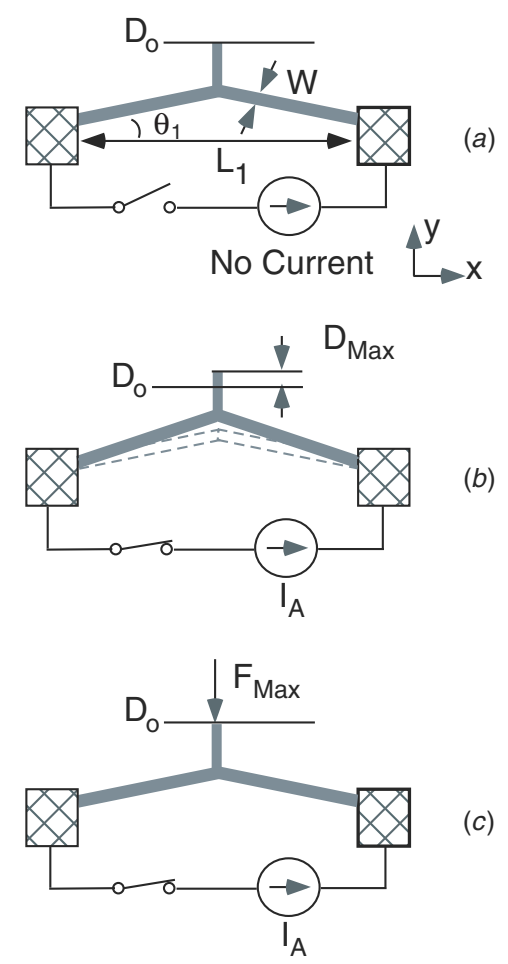

(a)

Figure 2. The actuation characteristics of a bent-beam actuator can be characterized with $D_{\max }$, the maximum unloaded displacement and $F_{\max }$, the maximum loading force.

the bent-beam results in mechanical strain due to thermal expansion, which is translated into mechanical displacement by the bent-beam structure. Its behavior can be described using three parameters: $D_{\max }$, the maximum unloaded displacement (figure 2(a)), $F_{\max }$, the maximum loading force that nullifies the unloaded displacement (figure $2(b)$ ) and the mechanical spring constant of the actuator, $K_{e q}$, which is typically the ratio of these. It can be expressed as [5]:

$$
K_{e q}=\frac{2 \sin ^{2}\left(\theta_{1}\right) W T E}{L_{1}}
$$

where $W$ is the width of the bent-beam, $L_{1}$ is the length, $T$ is the thickness, $\theta_{1}$ is the bending angle and $E$ is the Young's modulus of the material.

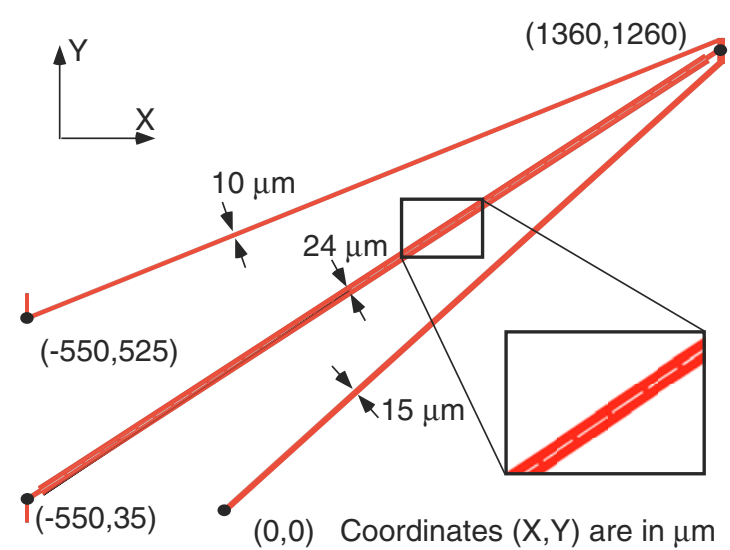

Figure 3. The dimensions for the compliant displacement amplifier.

\section{Actuator design}

In designing a $1 \mathrm{D}$ electrothermal actuator, one must consider the mechanical load connected to the actuator including the compliant displacement amplifier and the interconnect spring. For the 2D device, the shank connecting to the complementary actuator provides an additional load that can be substantial. The actuator design must be able to deliver sufficient force to reach the desired maximum tip displacement $\left(D_{q}\right)$, which is specified as $20 \mu \mathrm{m}$ in the design goals; this value was chosen partly because larger displacements can be easily accomplished with other positioning techniques. The loading force $\left(F_{q}\right)$ can also be found if the spring constant of the external mechanical load $\left(K_{L}\right)$ is identified. The operating point (or $Q$ point) at the highest power level can be specified with the values of $D_{q}$ and $F_{q}$ using $F_{q}=D_{q} K_{L}$ for a linear load. Among the external loading elements, the amplifier has the highest stiffness and is the most significant element.

The behavior of the displacement amplifier design is modeled using finite element analysis (FEA). The dimensions for these simulations are as indicated in figure 3 . The structural material is silicon with a Young's modulus of $160 \mathrm{GPa}$ and a thickness of $60 \mu \mathrm{m}$. Coventorware ${ }^{\mathrm{TM}}$ is used for the mechanical simulations described in this section. Both the displacement amplification factor and input spring constant are obtained by FEA using tetrahedral mesh elements. Stress stiffening does not play a significant role in the behavior of these microstructures and can be ignored for the displacements considered here. The amplification factor is the ratio of output and input displacements, while the input spring constant is the ratio of input force and input displacement. For the dimensions specified in figure 3 , the amplification factor under a $3 \mathrm{mN}$ force input is 3.17 and the input spring constant is $478.5 \mathrm{~N} \mathrm{~m}^{-1}$. The stiffness of the amplifier increases $6.6 \%$ when the input force is increased from $0 \mathrm{mN}$ to $9.0 \mathrm{mN}$. The change in compliance is due to non-linearity caused by the geometric change in the structure. As the structure is deformed, especially when the deformation is large, its kinematics are changed and this reflects on the amplification factor. This behavior is observed frequently in compliant mechanisms. The worst case stiffness is $500 \mathrm{~N} \mathrm{~m}^{-1}$ for the amplifier. A similar procedure is used to determine the stiffness of the interconnect spring $\left(0.15 \mathrm{~N} \mathrm{~m}^{-1}\right)$ and the shank 


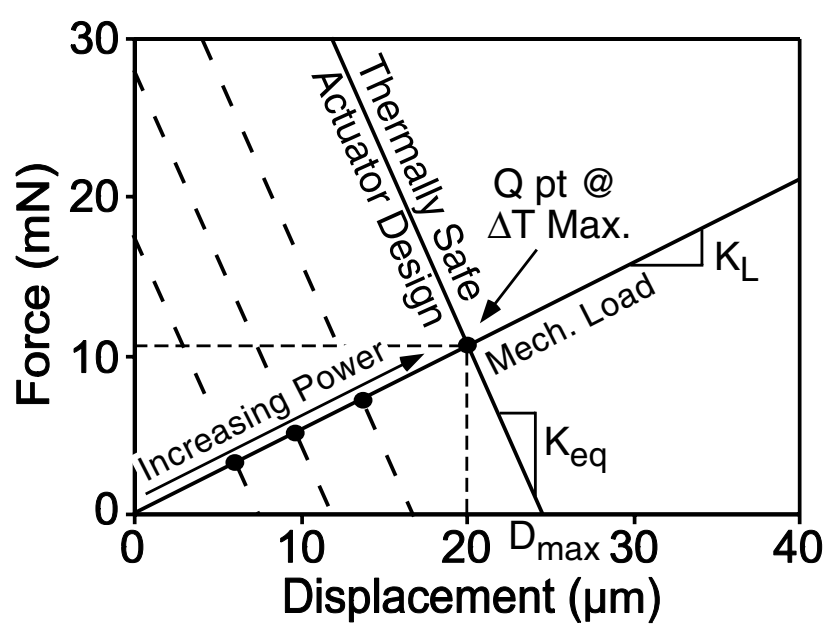

Figure 4. Load line analysis for determining the dimensions of the actuator.

$\left(29 \mathrm{~N} \mathrm{~m}^{-1}\right)$. The worst-case external mechanical load $\left(K_{L}\right)$ for the electrothermal actuator is $530 \mathrm{~N} \mathrm{~m}^{-1}$. This translates to a $F_{q}$ of $10.6 \mathrm{mN}$ at $20 \mu \mathrm{m}$ displacement.

A load line analysis is used to aid the design of the system (figure 4). The total mechanical load $\left(K_{L}\right)$ is represented by the line passing through the origin. The $Q$ point represents the design requirements for force and displacement specified with $D_{q}$ and $F_{q}$. Note that the $Q$ point represents the maximum operating point, i.e. the system will operate between the origin (where the actuator is unheated) and the $Q$ point (where the actuator is at its maximum heat load) along the $K_{L}$ line. The actuator design must be able to intercept the external load at the $Q$ point in order to satisfy the maximum displacement specifications; this means delivering $10.6 \mathrm{mN}$ at $20 \mu \mathrm{m}$ displacement. Also, it is crucial to keep the temperature in the actuator sufficiently low in order to avoid catastrophic actuator failures. In this case, $600{ }^{\circ} \mathrm{C}$ is used as the maximum average temperature increase allowed. This temperature is established from our previous work in silicon electrothermal actuators [5].

Another set of important design constraints arise from the physical layout of the device. The overall die size is fixed at $6 \mathrm{~mm} \times 6 \mathrm{~mm}$, which in turn limits the maximum length of the actuator, $L_{1}$, to $3000 \mu \mathrm{m}$. The thickness of the device, $T$, is constrained by the thickness of the structural layer in the fabrication process to $60 \mu \mathrm{m}$. This leaves the bending angle, $\theta_{1}$, and the width of the beam, $W$, as the two parameters used for actuator performance optimization. Design equations from $[5,8]$ are used to find a suitable angle and width to satisfy the $Q$ point without exceeding the maximum temperature allowed. Based on these constraints, a thermally safe actuator design is achieved: $L_{1}=3000 \mu \mathrm{m}, \theta_{1}=0.1 \mathrm{rad}, W=36 \mu \mathrm{m}$ and $T=$ $60 \mu \mathrm{m}$. The actuator has an internal stiffness of $2412 \mathrm{~N} \mathrm{~m}^{-1}$ and $F_{\max }=72.5 \mathrm{mN}$.

\section{Fabrication}

The micropositioner is fabricated from silicon-on-insulator (SOI) substrates made with fusion wafer bonding. Two wafers - one device wafer and one handle wafer-are cleaned and thermally oxidized to form $1.7 \mu \mathrm{m} \mathrm{SiO}_{2}$. The handle wafer is $540 \mu \mathrm{m}$ thick and the device wafer has an initial thickness of

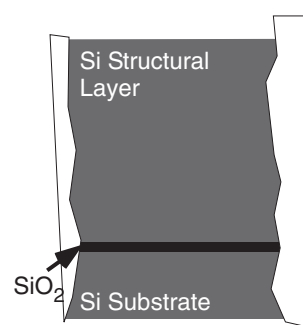

(a) SOI Wafer

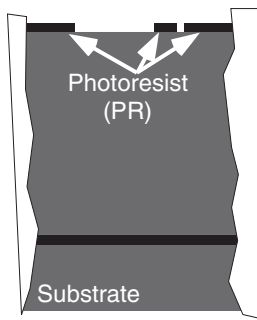

(b) PR Pattern

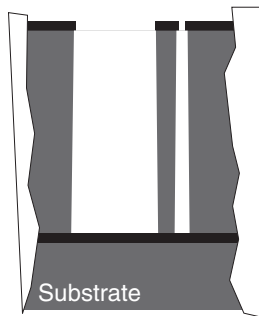

(c) DRIE Etch

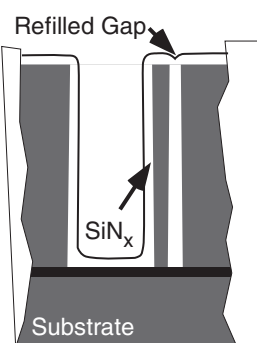

(d) Nitride Dep.

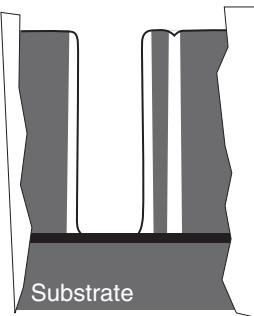

(e) Nitride Etch

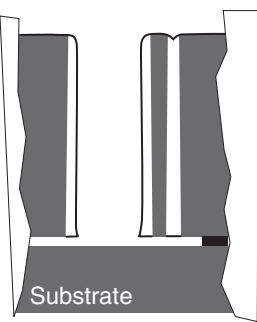

(f) Release
Figure 5. Fabrication of micropositioner using SOI substrates.

$650 \mu \mathrm{m}$. The resistivity of the handle wafer is not crucial, but the device layer is a highly conductive single crystal silicon ( 0.001 to $0.005 \Omega \mathrm{cm}$ ). The low resistivity device layer is necessary in order to provide suitable $V-I$ behavior for the electrothermal actuators. The two oxidized substrates are brought into contact, followed by a slow temperature ramp to $950{ }^{\circ} \mathrm{C}$ for fusion bonding. The bonded substrate is kept at high temperature for $1 \mathrm{~h}$ before a slow ramp down.

The device layer of the bonded SOI stack is thinned to the desired thickness by first grinding and polishing the device side to a thickness of $100 \mu \mathrm{m}$. Then, a $\mathrm{SF}_{6}$-based plasma in a parallel plate reactor is used to thin the stack to the final desired thickness. It was found that the surface of the device layer was acceptable for photolithography even after $4 \mathrm{~h}$ of dry etching (which resulted in up to $50 \mu \mathrm{m}$ of removal).

The SOI wafer is then patterned using a deep reactive ion etching (DRIE) step with $3.5 \mu \mathrm{m}$ of photoresist as masking material (figure 5(c)). Deep reactive ion etching uses the Bosch process [9], a fluorine-based chemistry that allows high-aspect ratio etches using alternating etch and sidewall passivation steps. Inductively coupled plasma (ICP) is used in this etch step to provide high plasma density while keeping the mean ion energy low, which allows the use of photoresist as a etch mask. After the etch terminates on the buried oxide layer, the wafer is cleaned and a $2.3 \mu \mathrm{m}$ layer of low-stress LPCVD nitride is deposited to form the electrical isolation using a trench refill process (figure $5(d)$ ); this step does not require extra masks. The excessive nitride is removed using a blanket etch (figure 5(e)), leaving nitride only on the sidewall 


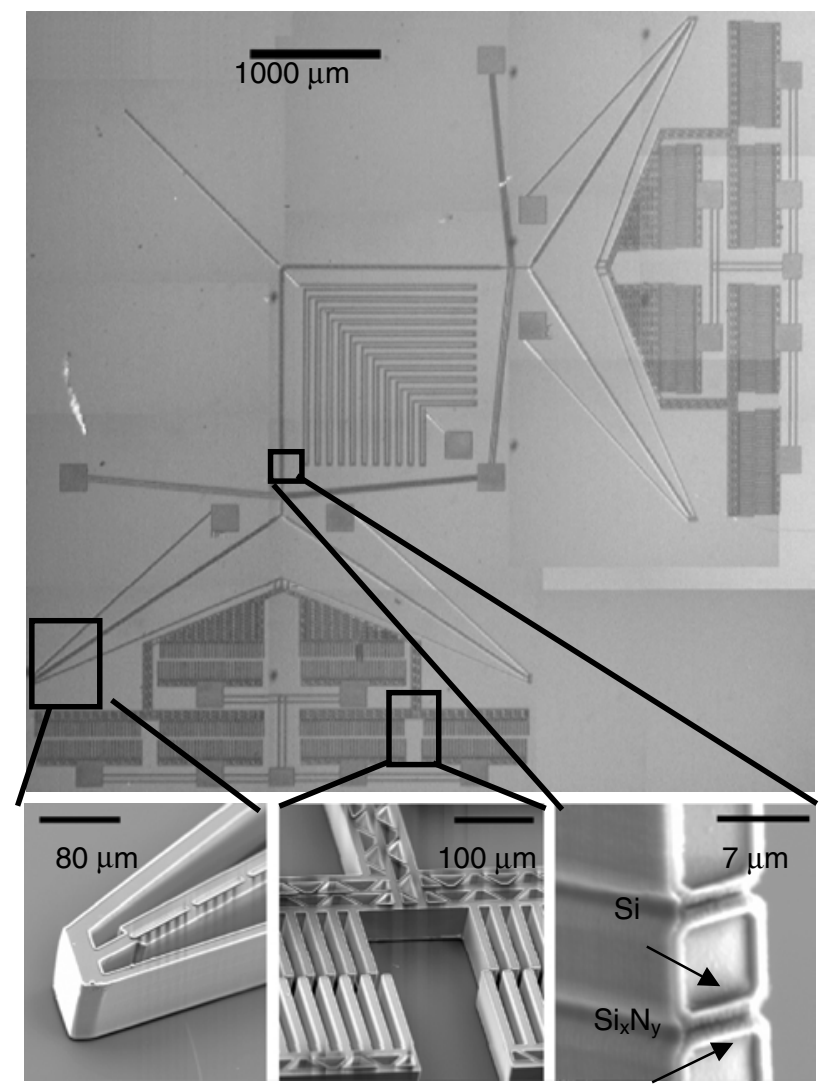

Figure 6. Optical image of positioner (above) and close-up SEM images of: the elbow microtransmission (left); capacitive tines (center) and $\mathrm{Si}_{x} \mathrm{~N}_{y}$ plugs (right).

of the structure and in the refilled plugs. This etch step was done in two stages: first, $80 \%$ of the thickness is removed with a $\mathrm{SF}_{6}$-based plasma, then, a $\mathrm{CHF}_{3}$ plasma is used for the removal of the remainder of the nitride. The latter gas is used because it has a selectivity of about 10:1 for nitride to silicon. The selectivity of nitride and oxide is also excellent. Finally, the device is released in 6:1 buffered oxide etchant (BOE) for $45 \mathrm{~min}$ and dried using a supercritical $\mathrm{CO}_{2}$ process (figure $5(f)$ ). Figure 6 shows the optical picture of the 2D positioner; the inserts show the sidewall profile of the structure. In the left insert, the elbow of the displacement amplifier is shown; the sidewall nitride layer can be seen as the layer which appears brighter than the silicon exposed at the top of the structure. Electrical isolation plugs are the result of the nitride refilling steps described earlier. A SEM of the plugs is shown in figure 6 .

\section{Experimental results}

The 1D operation of the structure is first verified by applying power to one of the actuators and monitoring the sensor capacitance (HP 4284A) in the actuation direction. At the same time, the capacitive position measurements are verified by optical displacement measurements taken with an imaging system. The results are shown in figure 7 . The tip displacement is the displacement measured at the input of the amplifier. The tine displacement is essentially the displacement seen by the capacitive sensor. A $15.1 \mu \mathrm{m}$ tip

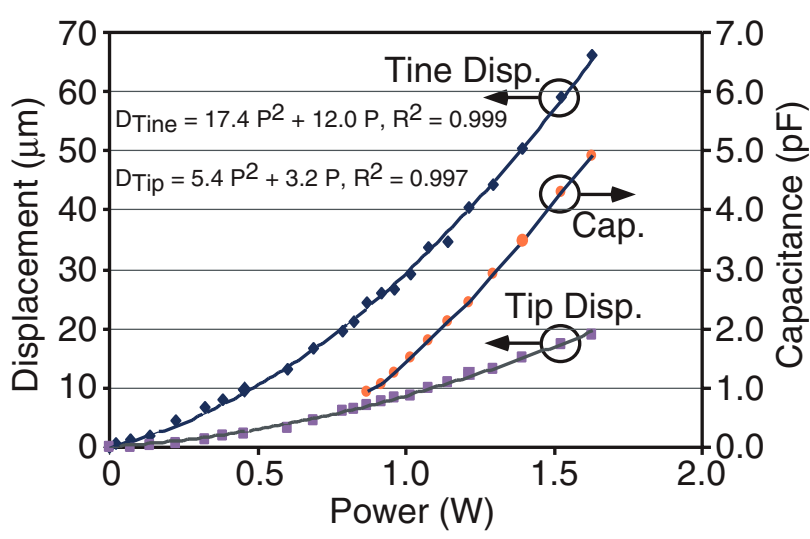

Figure 7. By applying power to one of the actuators, the capacitance of the sensor is measured simultaneously with the displacements.

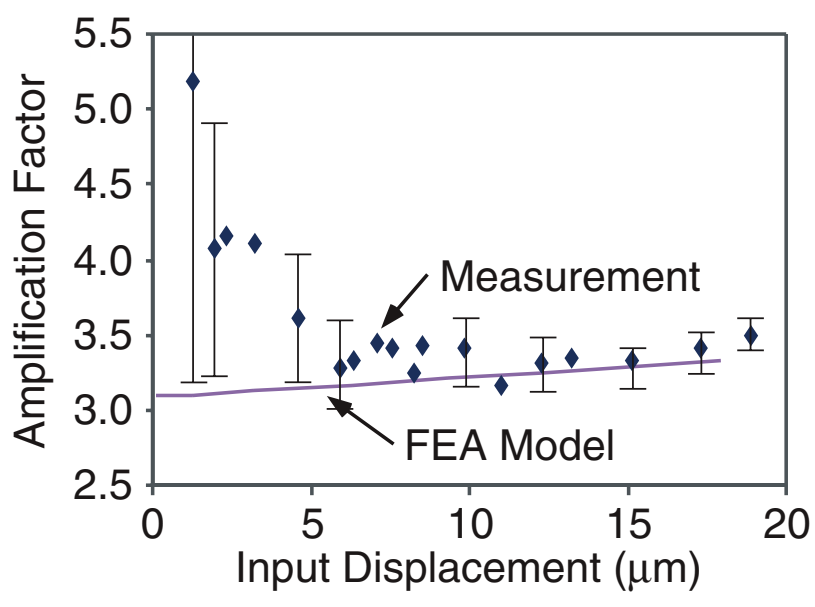

Figure 8. Measurement and modeling of mechanical amplification factor.

displacement requires $\approx 14 \mathrm{~V}$ and $100 \mathrm{~mA}$, and generates a tine displacement of $50.3 \mu \mathrm{m}$ and a capacitance change of $2.93 \mathrm{pF}$.

The measured amplification factor is found as the ratio of the tine displacement to the tip displacement, and is plotted in figure 8 alongside the FEA predicted values. The measured average amplification factor over input displacement of $5 \mu \mathrm{m}$ to $19 \mu \mathrm{m}$ is 3.37 , which compares well with the FEA predictions. The values of measured amplification factors have a large uncertainty for measurements with small tip displacement values $(<5 \mu \mathrm{m})$. This is due to the division of two small quantities with significant uncertainties. The calibrated imaging technique for displacement measurements has an accuracy of $\sim 500 \mathrm{~nm}$. The uncertainty is exaggerated when the measured tine displacement is divided by the small tip displacement with an uncertainty range in excess of 10 to $50 \%$ of the measured value. For example, at a tip displacement of $4.6 \pm 0.5 \mu \mathrm{m}$ and tine displacement of $16.6 \pm 0.5 \mu \mathrm{m}$, the uncertainties span from 3.16 to 4.17 . This measurement uncertainty is much reduced at larger displacements; for example, at $15 \mu \mathrm{m}$ input displacement, the uncertainty only ranges from 3.2 to 3.4 .

The measured sensitivity of the capacitive sensor is found as the ratio of the measured capacitance change divided by the input displacement change (measured using the calibrated 


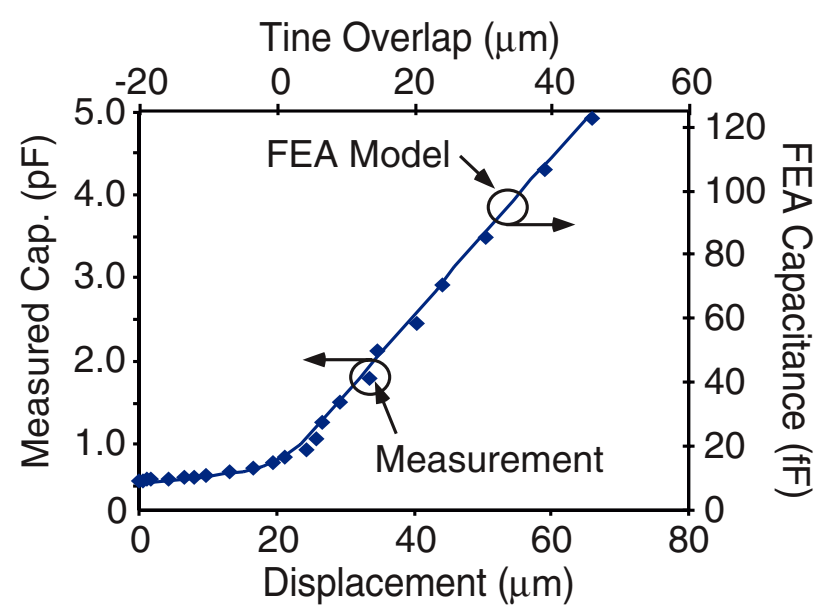

Figure 9. Measurement and modeling of the capacitance sensor bank response. The sidewall nitride refilling effect is used as a fitting factor.

imaging technique.) The capacitance measurements are taken with a high precision LCR meter (HP 4284A) which has an accuracy of $0.1 \mathrm{fF}$. Data points from input displacement values of $7 \mu \mathrm{m}$ to $19 \mu \mathrm{m}$ are used to find the sensitivity in the intended range of operation (with tines overlapping). The measured sensitivity for this mode of operation is $333.0 \pm 10 \mathrm{fF} \mu \mathrm{m}^{-1}$ averaged over the stated input range, or $0.333 \mathrm{fF} \mathrm{nm}^{-1}$. With an electrical set-up that can reliably measure $0.1 \mathrm{fF}$, which is routinely achievable using standard laboratory instruments, this sensitivity corresponds to a displacement accuracy of $0.3 \mathrm{~nm}$. If on-chip electronics are used, the displacement accuracy can be further improved. This measurement has a substantially lower uncertainty than the amplification factor measurement due to the high accuracy in the capacitance measurement and the reduced uncertainty in the input displacement in this range.

The measured capacitance data and the FEA modeling results are plotted in figure 9. The FEA model constructed contains only ten tine pairs for computational efficiency, although the actual number in the design is 174 pairs. Another factor considered during the fitting of the curves shown in figure 9 is the effect of the DRIE fabrication step on the capacitive gap size and the effect of silicon nitride on the sidewalls of the capacitive sensor; these two effects work against each other and both contribute to a scaling factor to the overall sensitivity. The effect of the DRIE step in the fabrication process works to widen the gaps between the tins in the sensor, and this reduces the sensitivity. After the etch step, the sidewalls are covered with silicon nitride, which has a higher permittivity than air, and serves to enhance the sensitivity. Their effects can be found using simple parallel plate models. For example, with no gap widening due to DRIE, capacitive sensitivity can be enhanced up to 3.9 times of the design value. In the fabricated device, it is estimated that the gaps between the capacitors are widened from the design value of $2 \mu \mathrm{m}$ to $4.6 \mu \mathrm{m}$ or $1.3 \mu \mathrm{m}$ erosion on each side of the gap. This kind of gap widening is very typical in such a process. The overall sensitivity modification is found to be 2.205 from both effects.

In any $2 \mathrm{D}$ positioner with orthogonally placed flexural drives, when one side is actuated, a small displacement is

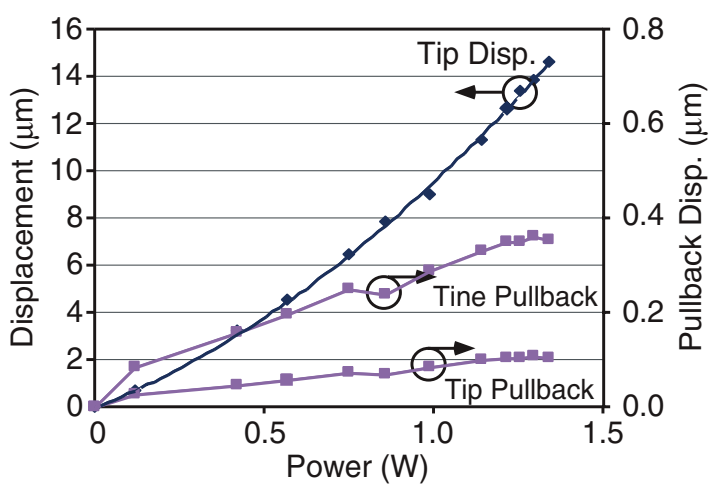

Figure 10. Power is applied to one axis, and the displacement error caused by this actuation at the other axis is measured and plotted.

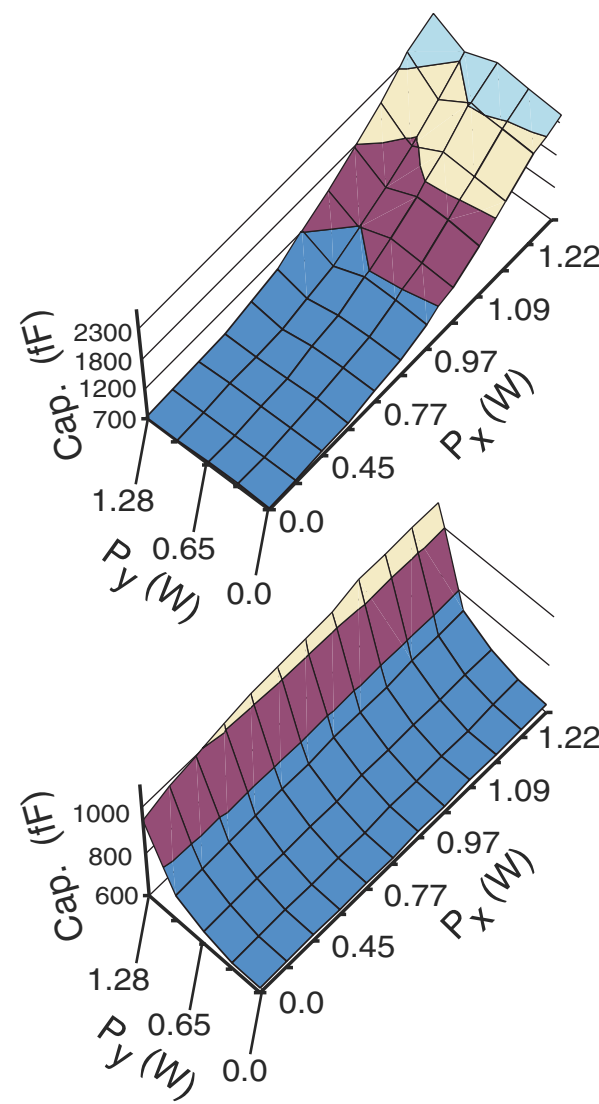

Figure 11. X-axis capacitance $(a)$ and Y-axis capacitance (b) plotted against input power levels in $\mathrm{X}$ and $\mathrm{Y}$ actuators.

geometrically imposed on the other one (figure 10). Although it is easy to null this by actuating the passive axis by a small amount, it is instructive to measure the uncompensated crosstalk. In this device the cross-talk (measured capacitively) corresponded to $105 \mathrm{~nm}$ for an actuated displacement of $14.6 \mu \mathrm{m}$, which is $\approx-43 \mathrm{~dB}$ of isolation. The fact that the cross-axial error is picked up by the capacitive sensor illustrates well that the structure can be operated to compensate for this effect.

The 2D operation of the device is demonstrated by actuating the two actuators simultaneously and measuring the capacitance change of both sensor banks. This result is shown in figure 11 . The shading in these two plots indicates 


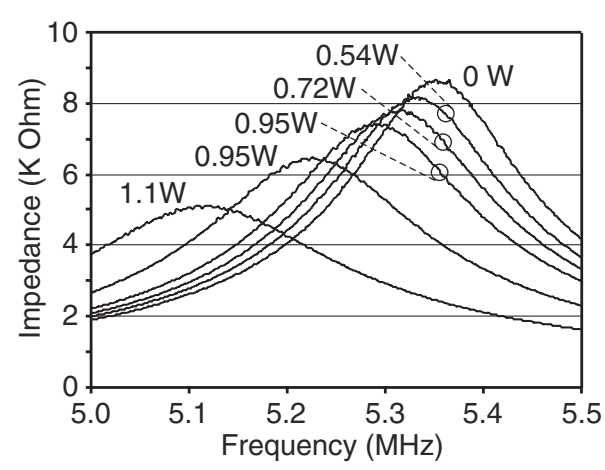

Figure 12. Applying the resonance method to capacitive position sensing.

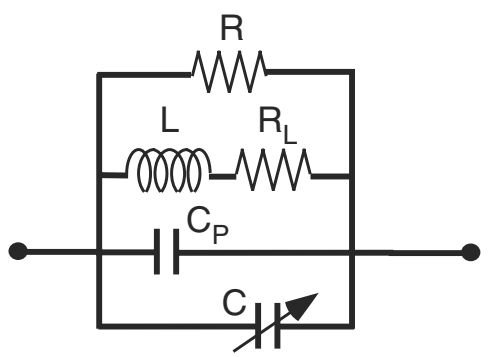

Figure 13. The electrical equivalent circuit for the resonance measurement.

the capacitance levels. Straight bands of shading would be considered ideal. Figure 11(a) shows the capacitance of the $\mathrm{X}$-axis sensor, while figure $11(b)$ shows the response of the Y-axis sensor. The non-idealities are primarily due to the cross-axial behavior just described.

Finally, the capacitance change in the sensor bank can be used in a simpler pickup technique, namely resonance shift method. By connecting a $100 \mu \mathrm{H}$ inductor in parallel with the capacitive sensor, one can measure the electrical resonance behavior of the structure. As the sense capacitance changes, the resonance peak shifts. This method is demonstrated in figure 12 , where it is clear that the resonance peak shifted $240 \mathrm{kHz}$ over $9.6 \mu \mathrm{m}$ of tip displacement. This $25 \mathrm{~Hz} \mathrm{~nm}^{-1}$ shift testifies to the high resolution capability of this device.

The shifting of the resonance frequency is consistent with the electrical behavior of the circuit model shown in figure 13, where $C$ is the sensor response, $L$ is the external inductor, $R_{L}$ is the series resistance in the inductor, $C_{P}$ is the parasitic capacitance and $R$ is the leakage resistance. The lowering of the resonance quality factor, $Q$, is attributed to series resistances in the measurement set-up and the external inductive element.

\section{Thermal-mechanical noise}

Thermal-mechanical noise (TMN) is a factor that can potentially limit the positioning resolution of the device. The effect is especially noticeable in systems with compliant mechanical suspensions such as electrostatic actuators. It arises from molecular agitation in and around the mechanical structure such as Brownian motion. The theoretical treatment of calculating TMN is detailed in [10] for a mass-spring- damper mechanical system. The thermal-mechanical noise force density, $F$, can be found using

$$
F=\sqrt{4 k_{\mathrm{B}} T D}
$$

where $k_{\mathrm{B}}$ is the Boltzmann's constant, $T$ is the absolute temperature and $D$ is the damping of the system; $F$ has a unit of $\left(\mathrm{N} \mathrm{H}_{z}^{-1 / 2}\right)$. In order to obtain the damping, $D$, the resonance frequency of the system is obtained using finiteelement analysis (FEA). The damping can be found using

$$
D=\frac{\sqrt{K M}}{Q_{\mathrm{M}}}
$$

where $K$ is the stiffness of the structure, $M$ is the mass and $Q_{\mathrm{M}}$ is the mechanical quality factor. Finally, the displacement due to TMN can be found using the following equation:

$$
X_{\mathrm{TM}-\mathrm{Noise}}=\frac{F \sqrt{f_{\text {res } 1}}}{K}=\frac{\sqrt{4 k_{\mathrm{B}} T D f_{\text {res } 1}}}{K}
$$

where $f_{\text {res1 }}$ is the frequency of the fundamental mode resonance.

Assuming a $Q_{\mathrm{M}}$ of $1-10$ (which is typical for such a system), the displacement due to TMN is calculated to be less than 1.1 picometer. This result indicates that TMN will not be a significant source of displacement error in this system.

\section{Conclusions}

A feedback controllable 2D positioner was designed, fabricated and tested. The unique construction allows the structure to achieve $19 \mu \mathrm{m}$ displacement at sub-nanometer resolution $(\sim 0.3 \mathrm{~nm})$. The capacitance change can be measured with standard laboratory instruments to provide subnanometer displacement accuracy. Also, using a parallel $L-C$ resonant circuit, a frequency shift of $240 \mathrm{kHz}$ is obtained over $9.6 \mu \mathrm{m}$ tip displacement, corresponding to a resolution of $25 \mathrm{~Hz} \mathrm{~nm}^{-1}$. Both methods are suitable for high resolution displacement measurements. The estimated positioning error due to thermal-mechanical noise is less than 1.1 picometer, which is well below the target resolution. Additional details regarding this project are described in [11].

\section{Acknowledgments}

The authors are grateful to Dr Joel Hetrick for the initial design of the displacement amplifier [7], and Dr Mo-huang Li for assistance with the temperature distribution measurement of the thermal actuators. This effort was supported in part by an NSF Career award to YG.

\section{References}

[1] Hirano T, Fan L-S, Lee W Y, Hong J, Semba T, Imaino W, Pattanaik S, Chan S and Webb P 1998 A micro-actuator for a hard-disk drive fine tracking servo Proc. ASME Intl. Mech. Eng. Congress \& Exposition (New York) pp 253-60

Hirano T, Fan L-S, Gao J Q and Lee W Y 1998 IEEE J. Microelectromech. Syst. 7 149-55

[2] Guckel H, Fischer K and Stiers E 1998 Closed loop controlled, large throw, magnetic linear microactuator with 1000 micron structural height Proc. IEEE Int. Conf. On Micro Electro Mechanical Systems pp 414-8 
[3] Rothuizen H, Drechsler U, Genolet G, Haberle W, Lutwyche M, Stutz R, Widmer R and Vettiger P 2000 Fabrication of a micromachined magnetic $\mathrm{X} / \mathrm{Y} / \mathrm{Z}$ scanner for parallel scanning probe applications Microelectron. Eng. 53 509-12

[4] Tuantranont A, Bright V M, Zhang J, Zhang W, Neff J and Lee Y C 2000 MEMS-controllable microlens array for beam steering and precision alignment in optical interconnect systems Proc. Solid-State Sensor and Actuator Workshop (Hilton Head) pp 101-4

[5] Que L, Park J-S and Gianchandani Y B 2001 Bent-beam electrothermal actuators: Part I. Single beam and cascaded devices IEEE/ASME J. Microelectromech. Syst. 10 247-54

[6] Chu L L and Gianchandani Y B 2002 A silicon micromachined, feedback controlled 2D micropositioner with sub-nanometer resolution Proc. Intl. Workshop on
Thermal Investigations of ICs and Systems (Madrid, Spain, Oct. 2002) pp 95-9

[7] Chu L L, Hetrick J A and Gianchandani Y B 2002 High amplification compliant microtransmissions for rectilinear electrothermal actuators Sensors Actuators A 97-98 776-83

[8] Gianchandani Y B and Najafi K 1996 Bent-beam strain sensors IEEE J. Microelectromech. Syst. 5 52-8

[9] Lärmer F and Schilp A 1996 Method of anisotropically etching silicon US Patent 5501893, German Patent DE4241045

[10] Gabrielson T B 1993 Mechanical-thermal noise in micromachined acoustic and vibration sensors IEEE Trans. Electron Devices 40 903-9

[11] Chu L L 2003 Feedback controllable 1D and 2D micro positioners using electro-thermal actuators and capacitive displacement sensors $P h D$ Thesis University of Wisconsin 